\title{
Expression Signatures of Long Noncoding RNAs in Adolescent Idiopathic Scoliosis
}

\author{
Xiao-Yang Liu, ${ }^{1,2}$ Liang Wang, ${ }^{1}$ Bin Yu, ${ }^{1}$ Qian-yu Zhuang, ${ }^{1}$ and Yi-Peng Wang ${ }^{1}$ \\ ${ }^{1}$ Department of Orthopedic Surgery, Peking Union Medical College Hospital, Chinese Academy of Medical Sciences \& \\ Peking Union Medical College, No. 1 Shuaifuyuan, Wangfujing, Dongcheng District, Beijing 100730, China \\ ${ }^{2}$ Department of Spine Surgery, Shandong Provincial Hospital Affiliated to Shandong University, Jinan, Shandong 250000, China
}

Correspondence should be addressed to Yi-Peng Wang; ypwang133@163.com

Received 4 June 2014; Accepted 31 October 2014

Academic Editor: Diego Gazzolo

Copyright (C) 2015 Xiao-Yang Liu et al. This is an open access article distributed under the Creative Commons Attribution License, which permits unrestricted use, distribution, and reproduction in any medium, provided the original work is properly cited.

\begin{abstract}
Purpose. Adolescent idiopathic scoliosis (AIS), the most common pediatric spinal deformity, is considered a complex genetic disease. Causing genes and pathogenesis of AIS are still unclear. This study was designed to identify differentially expressed long noncoding RNAs (lncRNAs) involving the pathogenesis of AIS. Methods. We first performed comprehensive screening of lncRNA and mRNA in AIS patients and healthy children using Agilent human lncRNA + mRNA Array V3.0 microarray. LncRNAs expression in different AIS patients was further evaluated using quantitative PCR. Results. A total of 139 lncRNAs and 546 mRNAs were differentially expressed between AIS patients and healthy control. GO and Pathway analysis showed that these mRNAs might be involved in bone mineralization, neuromuscular junction, skeletal system morphogenesis, nucleotide and nucleic acid metabolism, and regulation of signal pathway. Four lncRNAs (ENST00000440778.1, ENST00000602322.1, ENST00000414894.1, and TCONS_00028768) were differentially expressed between different patients when grouped according to age, height, classification, severity of scoliosis, and Risser grade. Conclusions. This study demonstrates the abnormal expression of lncRNAs and mRNAs in AIS, and the expression of some lncRNAs was related to clinical features. This study is helpful for further understanding of lncRNAs in pathogenesis, treatment, and prognosis of AIS.
\end{abstract}

\section{Introduction}

Adolescent idiopathic scoliosis (AIS), accounting for $90 \%$ of all idiopathic scoliosis (IS) cases, affects more than $2 \%$ of the pediatric population and results in more than 600,000 physician visits annually [1]. Approximately, $1.5 \%$ patients ultimately need surgical correction for their curvature [2]. However, the causes involved in AIS remain obscure.

Genetic twin studies and observation of familial aggregation have revealed significant genetic contribution to IS $[3,4]$. A genetic survey study reported that an overall risk of IS in first-degree relatives was up to $11 \%$, as compared to 2.4 and $1.4 \%$ in second- and third-degree relatives, respectively. These studies indicate that IS is an inheritable complex disease. Molecular studies found that some critical regions on chromosomes were potentially important for the occurrence of scoliosis $[5,6]$. Genome-wide association studies of AIS in case-control cohorts also located candidate susceptibility genes, and these findings indicate that single nucleotide polymorphisms are valuable for the AIS prognosis [7]. However, inconsistent outcomes were observed among studies concerning the candidate genes in AIS [8-11]. Totally, the inheritance mode and pathogenic gene of AIS is uncertain until today.

Recent technological advances reveal that a major portion of the genome is being transcribed and that protein-coding sequences only account for a minority of cellular transcriptional output. MicroRNAs are endogenously expressed noncoding transcripts, shorter than 20 nucleotides. MicroRNA silences gene expression by targeting specific mRNAs on the basis of sequence recognition [12]. Long noncoding RNAs (lncRNAs) are transcripts longer than 100 nucleotides. lncRNAs in most cases mirror the features of protein-coding 
TABLE 1: Detailed information of the four pairs of participants.

\begin{tabular}{|c|c|c|c|c|c|c|c|c|c|}
\hline Number & Group & Sex & Height & Age (years) & Illness history (years) & Menarche & Classification & Cobb angle $\left({ }^{\circ}\right)$ & Risser sign \\
\hline $\mathrm{A} 1$ & AIS & $\mathrm{F}$ & 155 & 13.7 & 1 & $\mathrm{~N}$ & PUMCIIc2 & 50 & 0 \\
\hline A2 & AIS & $\mathrm{F}$ & 167 & 15.9 & 2 & $\mathrm{Y}$ & PUMCIIc2 & 40 & 4 \\
\hline A3 & AIS & $\mathrm{F}$ & 158 & 15.0 & 1 & $\mathrm{Y}$ & PUMCIb & 44 & 5 \\
\hline A4 & AIS & $\mathrm{F}$ & 159 & 14.2 & 3 & $\mathrm{Y}$ & PUMCIIc1 & 44 & 3 \\
\hline N1 & NC & $\mathrm{F}$ & 163 & 17.9 & 0 & $\mathrm{Y}$ & - & 0 & 5 \\
\hline N2 & NC & $\mathrm{F}$ & 163 & 13.2 & 0 & $\mathrm{Y}$ & - & 0 & 3 \\
\hline N3 & NC & $\mathrm{F}$ & 158 & 12.3 & 0 & $\mathrm{~N}$ & - & 0 & 0 \\
\hline N4 & $\mathrm{NC}$ & F & 170 & 15.5 & 0 & $\mathrm{Y}$ & - & 0 & 4 \\
\hline
\end{tabular}

genes except that the former does not contain a functional open reading frame. A handful of studies have implicated that lncRNAs are involved in a variety of disease states [13] and altered lncRNA levels can result in aberrant expression of gene products that may contribute to many biological processes and human diseases [14-16]. Circulating levels of lncRNAs were reported to reflect local pathophysiological conditions $[17,18]$. Expression level of RNA in peripheral blood has been used to evaluate local circumstance of spine $[19,20]$. However, the expression of lncRNAs and their functions in AIS are still unknown.

In the present study, we measured lncRNAs expression in AIS patients using microarray and quantitative PCR (qPCR) analysis. Our results disclosed lncRNAs expression profiles and provide new information for further exploration of pathogenesis and prognosis of AIS.

\section{Materials and Methods}

2.1. Patients. Written informed consent was obtained from all participants. The study was approved by the Institutional Review Board of Chinese Academy of Medical Sciences and Peking Union Medical College Hospital. One hundred and twenty AIS patients (AIS) and twenty normal children (NC) were included in the study. Of these participants, four AIS patients and four children were used for microarray analysis. No significant difference was found between two groups in age, menarche, and Risser sign. Detailed information of the four pairs of participants is summarized in Table 1. The diagnosis of AIS was made only when other causes of scoliosis, including vertebral malformation, neuromuscular disorder, and syndromic disorders, were ruled out. Participants were screened with Adams' forward bend test. Cobb angle measured with a scoliometer was at least $10^{\circ}$ [21]. Peripheral blood from each subject was added 3 times volume of Trizol LS reagent (Amboin) and immediately stored at $-80^{\circ} \mathrm{C}$ until use.

2.2. RNA Extraction. Total RNA was extracted using Trizol LS reagent according to the instructions recommended by the manufacturer. The RNA purity and concentration were evaluated with NanoDrop ND-1000 spectrophotometer. RNA integrity was determined with $1 \%$ formaldehyde denaturing gel electrophoresis, which revealed a good quality (data not shown).
2.3. RNA Labeling and Hybridization. Double-stranded cDNAs (containing T7 RNA polymerase promoter sequence) were synthesized from $1 \mathrm{mg}$ total RNA according to the manufacturer's instructions (Capitalbio) and labeled with a fluorescent dye (Cy3-dCTP). Labeled cDNA was denatured at $95^{\circ} \mathrm{C}$ for $3 \mathrm{~min}$ in hybridization solution. Agilent human lncRNA + mRNA Array V3.0 was hybridized in an Agilent Hybridization Oven overnight at $42^{\circ} \mathrm{C}$ and washed with two consecutive solutions $\left(0.2 \% \mathrm{SDS}, 2 \mathrm{x}\right.$ SSC for $5 \mathrm{~min}$ at $42^{\circ} \mathrm{C}$, and $0.2 \mathrm{xSS}$ for $5 \mathrm{~min}$ at room temperature).

2.4. Microarray Analysis. The data from lncRNA + mRNA Array were used to analyze data summarization, normalization, and quality control using the GeneSpring software V11.5 (Agilent). The differentially expressed genes were selected if the change of threshold values was $\geq 2$ or $\leq-2$ folds and if Benjamini-Hochberg corrected $P$ values were $<0.05$. The data was normalized and hierarchically clustered with CLUSTER 3.0 software. The data were performed to be Tree Visualization with Java Treeview software (Stanford University School of Medicine, Stanford, CA, USA).

2.5. Construction of the Coding-Non-Coding Gene Coexpression (CNC) Network. The CNC network was constructed based on the correlation analysis between the differentially expressed lncRNAs and mRNAs. LncRNAs and mRNAs with Pearson correlation coefficients not less than 0.99 were selected to draw the network using open source bioinformatics software Cytoscape (Institute of Systems Biology in Seattle). In network analysis, red nodes represent the upregulated lncRNAs, dark blue nodes represent the downregulated lncRNAs, pink nodes represent the upregulated mRNAs and light blue nodes represent the downregulated mRNAs, circular nodes represent mRNAs, triangular nodes represent lncRNAs, dashed lines represent a positive correlation, and solid lines represent an inverse correlation.

2.6. qPCR. qPCR was performed using SYBR Premix Ex Taq on Thermal Cycler Dice TP800 instrument. Four lncRNAs (ENST00000440778.1, ENST00000602322.1, ENST00000414894.1, and TCONS_00028768), which revealed differentially expression, were evaluated in all participants. Total RNA $(2 \mu \mathrm{g})$ was reversely transcribed into cDNA using a PrimeScript RT reagent kit containing a gDNA Eraser (TaKaRa) according to the manufacturer's instructions. PCR 


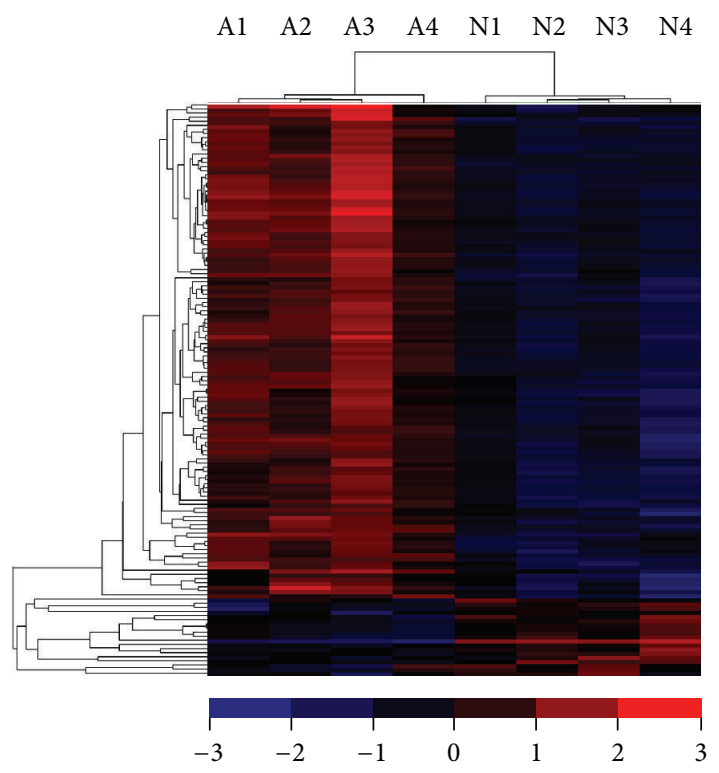

(a)

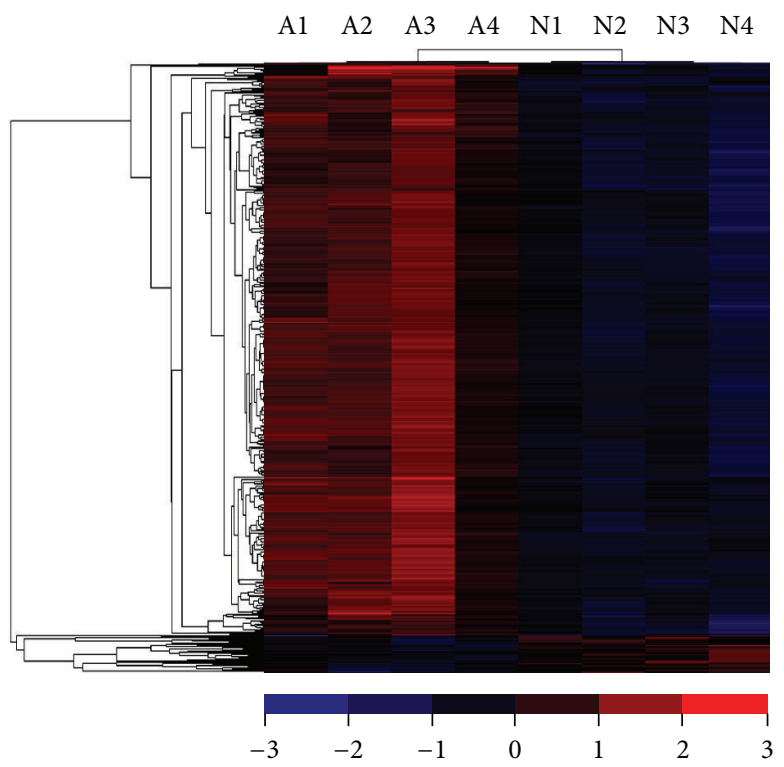

(b)

FIGURE 1: Heat maps of expression ratios (log2 scale) of lncRNAs (a) and mRNAs (b) between AIS patients and normal control. "Red" denotes high relative expression and "blue" denotes low relative expression.

TABLE 2: Primers used for qPCR.

\begin{tabular}{lccc}
\hline lncRNAs & Sense primer $\left(5^{\prime}-3^{\prime}\right)$ & Antisense primer $\left(5^{\prime}-3^{\prime}\right)$ & Product $(\mathrm{bp})$ \\
\hline ENST00000602322.1 & accttcccacctaccagtct & ctggggaaaccaccatagctt & 150 \\
ENST00000440778.1 & acttggttgcttttccccaca & gctttgggcttggaaagatgg & 107 \\
ENST00000414894.1 & agccgccctattcagttcac & tccaagttccgagttgtggg & 148 \\
TCONS_00028768 & gcaaacctggaaatctcggc & ggccgcagacatcatcttct & 142 \\
GAPDH & ctataaattgagcccgcagcc & gcgcccaatacgaccaaatc & 154 \\
\hline
\end{tabular}

was performed in $20 \mu \mathrm{L}$ of reaction system, including $10 \mu \mathrm{L}$ SYBR Premix Ex Taq $(2 \mathrm{x}), 0.4 \mu \mathrm{L}$ of PCR forward primer $(10 \mu \mathrm{M}), 0.4 \mu \mathrm{L}$ of PCR reverse primer $(10 \mu \mathrm{M}), 1 \mu \mathrm{L}$ of cDNA, and $8.2 \mu \mathrm{L}$ of double-distilled water. Primers for lncRNAs and mRNA are listed in Table 2. All experiments were performed in triplicates. All samples were normalized to GAPDH. The median in each triplicate was used to calculate relative lncRNAs concentrations $(\Delta \mathrm{Ct}=\mathrm{Ct}$ median lncRNAs-Ct median GAPDH). Folds change was calculated using $2^{-\Delta \Delta C t}$ methods. The differences of lncRNAs expression between patients and control were analyzed using Student's $t$ test within SPSS (version 16.0 SPSS Inc.). A value of $P<0.05$ was considered as statistically significant.

\section{Results}

3.1. LncRNAs Profiles. Tens of thousands lncRNAs were examined in microarray. A total of 139 lncRNAs revealed significantly different expression in AIS group, compared to NC group ( $\geq 2$-fold) (Table $S 1$, see Supplementary Material available online at http://dx.doi.org/10.1155/2015/276049). Compared to NC group, a total of 118 lncRNAs were consistently upregulated or downregulated in all tested AIS samples.
ENST00000440778.1 was the most significantly downregulated lncRNA (fold change $=9.780566$ ), while NR_024075 (Log2 fold change $=3.8194413$ ) was the most significantly upregulated one. The heat maps of the expression ratios of lncRNAs are shown in Figure 1(a).

3.2. mRNAs Profiles. The expression of coding transcripts (i.e., mRNAs) was examined with microarray containing 33,982 coding transcripts probes. Up to $546 \mathrm{mRNAs}$ showed significant difference between AIS and NC groups (Table S2). A total of 512 mRNAs were upregulated in AIS, while 34 mRNAs were downregulated. The heat map of the expression ratios of mRNAs is shown in Figure 1(b). GO analysis showed that these mRNAs might be involved in bone mineralization, neuromuscular junction, skeletal system morphogenesis, and nucleotide and nucleic acid metabolism. Pathway analysis indicates that the dysregulated mRNAs are involved in cell adhesion molecules, Wnt signaling pathway, Toll-like receptor signaling pathway, MAPK signaling pathway, and so on. Coding RNAs related to major biological processes are listed in Table 3. These results support the viewpoint that AIS may be a genetic disease involving musculoskeletal system. 
TABLE 3: Genes biological processes.

\begin{tabular}{|c|c|c|c|c|}
\hline Biological processes & Gene symbol & Gene name & Entrez gene ID & Fold changes \\
\hline \multirow{10}{*}{$\begin{array}{l}\text { Nervous system } \\
\text { development }\end{array}$} & LTA & "Phosphoinositide-3-kinase, catalytic, delta polypeptide" & 5293 & 2.1513674 \\
\hline & TP53 & Tumor protein $\mathrm{p} 53$ & 7157 & 2.0308032 \\
\hline & $\mathrm{MSH} 2$ & "mutS homolog 2, colon cancer, nonpolyposis type 1 (E. coli)" & 4436 & 2.0266845 \\
\hline & BCL2 & BCL2-associated athanogene 3 & 9531 & 2.130905 \\
\hline & TP53 & Tumor protein $\mathrm{p} 53$ & 7157 & 2.0308032 \\
\hline & NCL & Nuclear RNA export factor 1 & 10482 & 2.00108 \\
\hline & PAFAH1B1 & $\begin{array}{l}\text { "Platelet-activating factor acetylhydrolase } 1 b \text {, regulatory subunit } 1 \\
(45 \mathrm{kDa}) \text { " }\end{array}$ & 5048 & 2.1079702 \\
\hline & OLIG1 & Oligodendrocyte transcription factor 1 & 116448 & 2.129439 \\
\hline & PKD1 & Polycystic kidney disease 1 (autosomal dominant) & & 2.18849 \\
\hline & BTG2 & "BTG family, member 2" & 7832 & 2.7636645 \\
\hline \multirow{6}{*}{$\begin{array}{l}\text { Skeletal morphogenesis } \\
\text { and development }\end{array}$} & BCL2 & B-Cell CLL/lymphoma 2 & 596 & 2.252202 \\
\hline & IL6ST & "Interleukin 6 signal transducer (gp130, oncostatin M receptor)" & 3572 & 2.2222419 \\
\hline & MYC & v-myc myelocytomatosis viral oncogene homolog (avian) & 4609 & 2.768515 \\
\hline & TPP1 & Tripeptidyl peptidase I & 1200 & 2.0187664 \\
\hline & AES & Aminoterminal enhancer of split & 166 & 2.2728803 \\
\hline & ALOX15 & Arachidonate 15-lipoxygenase & 246 & 2.1062436 \\
\hline \multirow{11}{*}{$\begin{array}{l}\text { Muscle development } \\
\text { and function }\end{array}$} & BCL2 & B-Cell CLL/lymphoma 2 & 596 & 2.252202 \\
\hline & FLI1 & Friend leukemia virus integration 1 & 2313 & 2.2254617 \\
\hline & UTRN & Utrophin & 7402 & 2.1682687 \\
\hline & UTS2 & Urotensin 2 & 10911 & 2.469184 \\
\hline & HIF1A & $\begin{array}{l}\text { "Hypoxia inducible factor 1, alpha subunit (basic helix-loop-helix } \\
\text { transcription factor)" }\end{array}$ & 3091 & 2.028285 \\
\hline & TGFBR2 & "Transforming growth factor, beta receptor II (70/80 kDa)" & 7048 & 2.1115046 \\
\hline & TTN & Titin & 7273 & 2.7300012 \\
\hline & LEF1 & Lymphoid enhancer-binding factor 1 & 51176 & 3.1550286 \\
\hline & HIF1A & $\begin{array}{l}\text { "Hypoxia inducible factor 1, alpha subunit (basic helix-loop-helix } \\
\text { transcription factor)" }\end{array}$ & 3091 & 2.028285 \\
\hline & FAM65B & "Family with sequence similarity 65, member B" & 9750 & 2.247876 \\
\hline & UTRN & Utrophin & 7402 & 2.1682687 \\
\hline \multirow{3}{*}{$\begin{array}{l}\text { Notch signaling } \\
\text { pathway }\end{array}$} & ADAM17 & ADAM metallopeptidase domain 17 & 6868 & 2.0420005 \\
\hline & $\mathrm{NOTCH} 2$ & Notch 2 & 4853 & 2.1405845 \\
\hline & CTBP1 & C-Terminal binding protein 1 & 1487 & 2.050679 \\
\hline \multirow{13}{*}{$\begin{array}{l}\text { MAPK signaling } \\
\text { pathway }\end{array}$} & TAB2 & TGF-beta activated kinase 1/MAP3K7 binding protein 2 & 23118 & 2.1040483 \\
\hline & SOS1 & Son of sevenless homolog 1 (Drosophila) & 6654 & 2.0901704 \\
\hline & DUSP16 & Dual specificity phosphatase 16 & 80824 & 2.1876025 \\
\hline & MAP3K1 & Mitogen-activated protein kinase kinase kinase 1 & 4214 & 2.343032 \\
\hline & RPS6KA3 & "Ribosomal protein S6 kinase, $90 \mathrm{kDa}$, polypeptide 3" & 6197 & 2.0745454 \\
\hline & CD14 & CD14 molecule & 929 & 2.3363564 \\
\hline & DUSP6 & Dual specificity phosphatase 6 & 1848 & 2.2230558 \\
\hline & ARRB1 & "Arrestin, beta 1" & 408 & 2.004528 \\
\hline & TGFBR2 & “Transforming growth factor, beta receptor II (70/80 kDa)” & 7048 & 2.1115046 \\
\hline & AKT3 & $\begin{array}{l}\text { "v-akt murine thymoma viral oncogene homolog } 3 \text { (protein kinase } \\
\text { B, gamma)" }\end{array}$ & 10000 & 2.0013347 \\
\hline & MYC & v-myc myelocytomatosis viral oncogene homolog (avian) & 4609 & 2.768515 \\
\hline & TP53 & Tumor protein p53 & 7157 & 2.0308032 \\
\hline & NFKB1 & Nuclear factor of kappa light polypeptide gene enhancer in B-cells 1 & 4790 & 2.0809784 \\
\hline
\end{tabular}


TABLE 3: Continued.

\begin{tabular}{|c|c|c|c|c|}
\hline Biological processes & Gene symbol & Gene name & Entrez gene ID & Fold changes \\
\hline \multirow{15}{*}{ Wnt signaling pathway } & CAMK2G & Calcium/calmodulin-dependent protein kinase II gamma & 818 & 2.1046312 \\
\hline & TCF7 & “Transcription factor 7 (T-cell specific, HMG-box)” & 6932 & 2.6018746 \\
\hline & PPARD & Peroxisome proliferator-activated receptor delta & 5467 & 2.1376982 \\
\hline & TCF3 & “Transcription factor 7 (T-cell specific, HMG-box)" & 6932 & 2.8202255 \\
\hline & CAMK2D & Calcium/calmodulin-dependent protein kinase II delta & 817 & 2.5258021 \\
\hline & PLCB2 & "Phospholipase C, beta 2" & 5330 & 2.2920265 \\
\hline & NFATC1 & $\begin{array}{l}\text { "Nuclear factor of activated T-cells, cytoplasmic, } \\
\text { calcineurin-dependent 1" }\end{array}$ & 4772 & 2.0340881 \\
\hline & MYC & v-myc myelocytomatosis viral oncogene homolog (avian) & 4609 & 2.768515 \\
\hline & TP53 & Tumor protein p53 & 7157 & 2.0308032 \\
\hline & LEF1 & Lymphoid enhancer-binding factor 1 & 51176 & 3.1550286 \\
\hline & CTBP1 & C-Terminal binding protein 1 & 1487 & 2.050679 \\
\hline & PIAS4 & "Protein inhibitor of activated STAT, 4" & 51588 & 2.0654333 \\
\hline & AES & Aminoterminal enhancer of split & 166 & 2.2728803 \\
\hline & INVS & Inversin & 27130 & 2.2433844 \\
\hline & LRRFIP2 & Leucine rich repeat (in FLII) interacting protein 2 & 9209 & 2.1049194 \\
\hline
\end{tabular}

Gene Ontology and Pathway analysis was performed for differentially expressed mRNAs. mRNAs involving musculoskeletal development are listed in their gene groups with associated Entrez Gene ID and fold changes per gene.

TABLE 4: Correlation between lncRNAs and mRNAs.

\begin{tabular}{lcccccr}
\hline Source $^{\mathrm{a}}$ & lncRNA & mRNA & Gene symbol & Correlation & $P$ value $^{\text {cis-Regulation }^{\mathrm{b}}}$ \\
\hline p33784 & uc002ddj.1 & A_21_P0011418 & PKD1 & 0.9967847 & $8.29 E-08$ & Sense \\
p33788 & uc021tnw.1 & A_24_P282108 & ZZEF1 & 0.9976724 & $3.15 E-08$ & Sense \\
p26337 & uc021zdc.1 & A_23_P122615 & PNISR & 0.9907335 & $1.98 E-06$ & Sense \\
p26595 & HIT000067310 & A_23_P391275 & RCAN3 & 0.9967367 & $8.67 E-08$ & Intergenic \\
p112 & ENST00000577528.1 & A_23_P391275 & RCAN3 & 0.9965706 & $1.01 E-07$ & Intergenic \\
p2444 & ENST00000602322.1 & A_33_P3210139 & PCF11 & 0.9932224 & $7.74 E-07$ & Antisense \\
p26595 & HIT000067310 & A_23_P35205 & RCAN3 & 0.9900216 & $2.47 E-06$ & Sense \\
p29552 & TCONS_00001429 & A_23_P35205 & RCAN3 & 0.9956033 & $2.12 E-07$ & Antisense \\
\hline
\end{tabular}

${ }^{a}$ Probe name of lncRNA. ${ }^{b}$ sense: the lncRNAs is a coding transcript exon on the same genomic strand; antisense: the lncRNA is transcribed from the antisense strand; intergenic: there are no overlapping or bidirectional coding transcripts nearby the lncRNA within $10 \mathrm{kbp}$.

3.3. CNC Network. In the CNC network, there were 76 lncRNAs and 370 mRNAs. A total of 446 network nodes were gained and this was associated with 1121 pairs of coexpression of lncRNAs and mRNAs. The CNC network indicates that one mRNA is correlated with one to tens of lncRNAs and vice versa. The whole $\mathrm{CNC}$ network might implicate an interregulation between lncRNAs and coding RNAs in AIS. In particular, lncRNA ENST00000602322.1 was correlated with lncRNA ENST00000422231.2 and nine coding RNAs (Figure 2).

More and more evidences indicate that lncRNAs play important roles in gene expression. Thus, we explored the correlation between lncRNAs and mRNA. More than 1000 pairs of lncRNAs and mRNAs reached an absolute correlation coefficient greater than 0.99 and a False Discovery Rate less than 0.01. Target prediction indicated that seven lncRNAs (uc002ddj.1, uc021tnw, uc021zdc.1, HIT000067310, ENST00000577528.1, ENST00000602322.1, and TCONS_00001429) may influence the expression of related mRNAs. ENST00000602322.1 and HIT000067310 may regulate the mRNA expression of PCF11 and RCAN3, respectively. The two mRNAs are expressed in muscle system and encode proteins which regulate mRNA splicing and metabolism. These lncRNAs and mRNAs may play a role in the pathogenesis of AIS. Detailed information of $\operatorname{lncRNAs}$ and predicted mRNAs is listed in Table 4.

3.4. LncRNA Classification and Subgroup Analysis. Recently, some special classes or 14 clusters of lncRNAs have been identified with specific function in human cells. Numerous lncRNAs were found to be transcribed from the human homeobox transcription factors (HOX) clusters [22]. These lncRNAs are expressed in temporal and site-specific fashions and may be associated with distinct and diverse biological processes [23, 24], such as cell proliferation, RNA binding complexes, and muscle development. In the present study, our microarray probes targeted 407 discrete transcribed regions of four human HOX loci. None of them was differentially expressed between AIS and NC. 


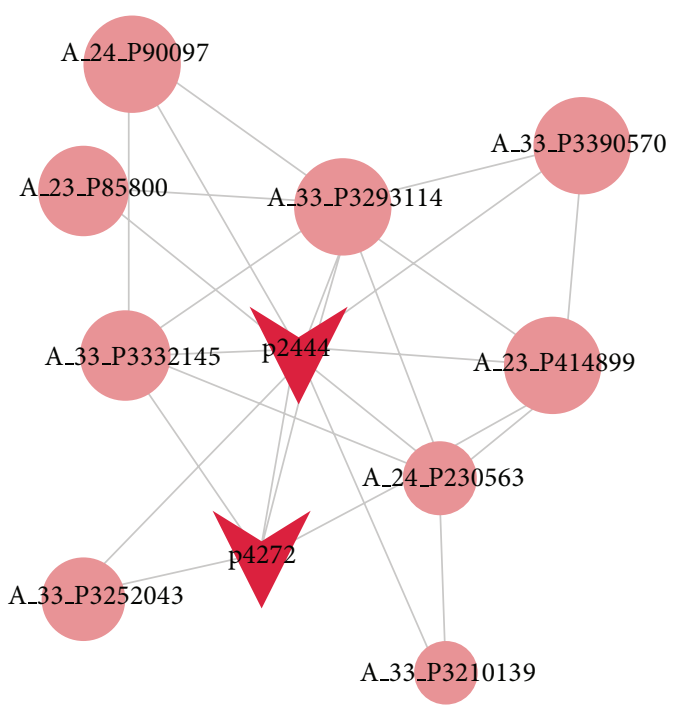

Figure 2: CNC network of lncRNA ENST00000602322.1 (labeled as p2444). Circular nodes represent mRNA; triangular nodes represent lncRNA. Detailed information of lncRNAs and mRNAs is listed in supplementary tables.

Harrow and his colleagues defined a set of $\operatorname{lncRNAs}$ with enhancer-like function in human cell lines [25]. Depletion of these lncRNAs led to decreased expression of their neighboring protein-coding genes. In the present study, 1896 lncRNAs with enhancer-like function were detected while 4 of them were differentially expressed in AIS. The differentially expressed enhancer-like lncRNAs and their nearby coding genes (distance, $300 \mathrm{~kb}$ ) are shown in Table S3.

3.5. $q P C R$ Validation. Data from microarray were validated by $\mathrm{qPCR}$ in twenty pairs of samples. qPCR results revealed that ENST00000602322.1, ENST00000414894.1, and TCONS_00028768 were upregulated and ENST00000440778.1 was downregulated in AIS samples $(P<0.01)$, as compared to the control, which was consistent with those from microarray analysis (Figure 3).

3.6. Clinical Features of Validated lncRNAs. The correlation of lncRNA expression with clinical features was further analyzed. All 120 AIS patients were grouped according to age, height, menarche, classification, severity of scoliosis (measured by Cobb angle), and Risser grade (Table 5). No significant difference of $\ln c \mathrm{RNAs}$ expression was observed when patients were grouped according to menarche. The expression of ENST00000440778.1 and TCONS_00028768 was significantly different when grouped according to height $(P<0.05)$. The expression of ENST00000602322.1 was higher in younger patients $(P<0.01)$. Significant difference was also observed in ENST00000602322.1 expression between patients with single curve and double curves $(P<0.05)$. Lower expression of ENST00000414894.1 was observed in patients with Cobb angle greater than $40^{\circ}(P<0.05)$. The expression of ENST00000440778.1 was higher in patients with Risser grade $\leq 3(P<0.05)$.

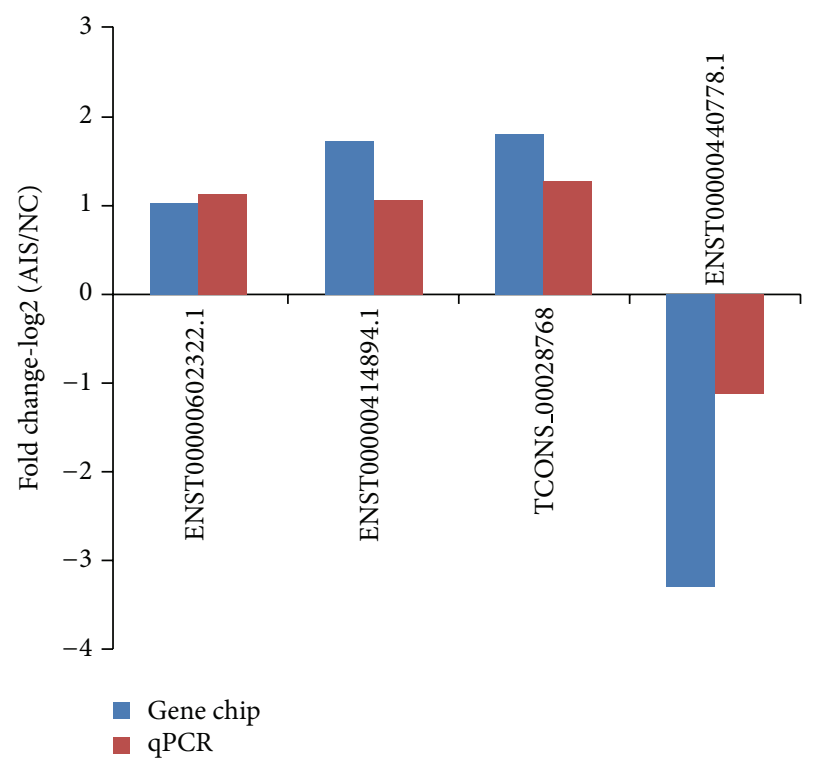

FIGURE 3: Comparison between microarray and qPCR results. The columns heights represent the log-transformed median fold changes (AIS/NC) in the AIS patients compared to normal control. The validation results of the four lncRNAs indicate that the microarray results match well the qPCR results.

\section{Discussion}

Although many clinical, epidemiological, and basic science researches were performed, the etiopathogenesis of AIS is still unclear [26]. Over the past decades, significant achievements have been made in profiling the molecular signatures in diseases using gene expression microarray [27]. Microarray has been recognized as a feasible and useful approach to explore pathogenesis and genetics of diseases and seek specific biomarkers [28]. Recently, hundreds of lncRNAs have been discovered, and altered lncRNAs expression has been considered to be correlated with cancer pathogenesis and muscles differentiation $[29,30]$. However, the potential role of lncRNA expression in etiology and pathogenesis of AIS has not been systematically investigated.

To uncover the expression pattern of lncRNAs in AIS, we investigated the lncRNA expression signatures using microarray and found hundreds of differentially expressed $\operatorname{lncRNAs}$ in AIS patients. These lncRNAs may be involved in the development and progression of AIS. Based on the previous work and computer analysis, four lncRNAs (ENST00000440778.1, ENST00000602322.1, ENST00000414894.1, and TCONS_00028768) were selected to validate the consistency using qPCR. The expression of ENST00000440778.1 was downregulated in microarray and qPCR. But the expression gap between AIS and normal control was narrower in qPCR than in microarray. The difference may result from selection bias. Samples used in microarray analysis may lack representativeness, and ENST00000440778.1 expression in microarray analysis was not completely coincident between participants. Besides, it is necessary to investigate the specific lncRNAs in bigger size of samples. 
TABLE 5: Expressions of lncRNAs in different clinical features.

\begin{tabular}{lccccc}
\hline \multicolumn{1}{c}{ Features } & & ENST00000602322.1 & ENST00000440778.1 & ENST00000414894.1 & TCONS_00028768 \\
\hline \multirow{2}{*}{ Menarche age $(\mathrm{y})$} & $\leq 11$ & $7.29 \pm 0.82$ & $4.74 \pm 0.88$ & $3.04 \pm 0.52$ & $5.51 \pm 1.08$ \\
& $>11$ & $7.75 \pm 1.20$ & $5.09 \pm 1.25$ & $2.95 \pm 0.75$ & $5.62 \pm 1.44$ \\
\hline \multirow{2}{*}{ Height $(\mathrm{cm})$} & $\leq 160$ & $7.49 \pm 1.03$ & $4.40 \pm 0.71^{*}$ & $2.98 \pm 0.59$ & $5.05 \pm 0.99^{*}$ \\
& $>160$ & $7.73 \pm 1.17$ & $5.34 \pm 1.45^{*}$ & $2.99 \pm 0.78$ & $5.95 \pm 1.63^{*}$ \\
\hline \multirow{2}{*}{ Onset of AIS $(\mathrm{y})$} & $\leq 12$ & $6.73 \pm 0.60^{* *}$ & $4.92 \pm 1.09$ & $3.09 \pm 1.52$ & $5.54 \pm 1.19$ \\
& $>12$ & $7.86 \pm 1.29^{* *}$ & $5.01 \pm 1.17$ & $2.87 \pm 1.17$ & $5.63 \pm 1.43$ \\
\hline \multirow{2}{*}{ Numbers of curves ${ }^{\mathrm{a}}$} & 1 & $7.09 \pm 0.74^{*}$ & $4.95 \pm 1.40$ & $2.79 \pm 0.66$ & $5.81 \pm 1.24$ \\
& 2 & $7.98 \pm 1.19^{*}$ & $4.98 \pm 0.95$ & $3.10 \pm 0.66$ & $5.46 \pm 1.34$ \\
\hline \multirow{2}{*}{ Cobb angle $\left.{ }^{\circ}\right)$} & $\leq 40$ & $7.60 \pm 1.36$ & $5.11 \pm 1.70$ & $2.47 \pm 0.63^{*}$ & $5.59 \pm 1.60$ \\
& $>40$ & $7.58 \pm 1.95$ & $4.90 \pm 0.72$ & $2.93 \pm 0.60$ & $5.59 \pm 1.15$ \\
\hline \multirow{2}{*}{ Risser sign ${ }^{\mathrm{b}}$} & $\leq 3$ & $7.51 \pm 1.12$ & $4.33 \pm 0.81^{*}$ & $5.02 \pm 0.72$ & $5.37 \pm 0.97$ \\
& $>3$ & $7.65 \pm 1.07$ & $5.21 \pm 1.25^{*}$ & $5.76 \pm 1.51$ \\
\hline
\end{tabular}

Relative lncRNAs levels were normalized to GAPDH $(\triangle \mathrm{Ct}=\mathrm{CtlncRNA}-\mathrm{CtGAPDH})$. Results are presented as mean \pm standard deviation. ${ }^{*} P<0.05,{ }^{* *} P<$ 0.01 . ${ }^{\mathrm{a}}$ Curves were counted and classified into single, double, and triple curves according to the apex number. ${ }^{\mathrm{b}}$ Risser sign refers to the amount of calcification of the human pelvis as a measure of maturity. On a scale of 5 , it gives a measurement of ossification progression; the grade of 5 means that skeletal maturity is reached.

In the present study, the differentially expressed mRNAs in AIS patients involve musculoskeletal development processes, including bone mineralization, neuromuscular junction, skeletal system morphogenesis, and nucleotide and nucleic acid metabolism. Pathway analysis indicates that the dysregulated mRNAs are related to cell adhesion molecules, Wnt signaling pathway, Toll-like receptor signaling pathway, MAPK signaling pathway, and so on. Precious studies have revealed the relationship between synapse formation, bone mineralization, and Wnt signaling pathway [31-33]. MAPK signaling pathway was also reported to be related to osteoblast differentiation and intervertebral disc cells degeneration [34, 35]. These biological processes and signaling pathway may play a significant role in the musculoskeletal system and pathogenesis of AIS.

More and more evidences reveal that lncRNAs act in both cis and trans [36, 37]. LncRNA ENST00000602322.1 locates on chromosome 11q and is adjacent to Pcfll (Protein 1/Cleavage Factor 1). Pcfl1 participates in transcription by coupling pre-mRNA [38]. Pcfll is also found to play a role in transcription initiation, elongation, and mRNA export from nucleus to cytoplasm [39-41]. Given the key role of Pcfll in transcription, it may not be surprising that ENST00000602322.1 may relate to the pathogenesis of AIS. The more detailed mechanisms of ENST00000602322.1 in transcription and translation need further investigation.

ENST00000440778.1 was upregulated 9.78-fold in AIS. Its expression was even higher in each AIS patient than that in healthy participants. Little is known about the function of ENST00000440778.1. However, its potential role in AIS pathogenesis was implied not only by its expression change but also by the clinical data from different height and Risser sign.

Our findings demonstrate differential lncRNA expression patterns in AIS when grouped according to different clinical features. This suggests potential significance in treatment and prognosis evaluation. Clinically, classification and curvature angle are important in evaluating surgical treatment and making operation plan in AIS [42, 43]. Onset time and Risser grade also play important roles in evaluating the progression of scoliosis [44]. It has been reported that lncRNAs are more specific than protein-coding mRNAs [45] and are easier to be detected in the blood samples of neoplastic and nonneoplastic patients using conventional PCR method $[17,18]$. The differential expression of lncRNAs is potentially valuable in development of specific PCR markers and in providing more support on treatment and prognosis.

Several limitations exist in this study. First, although the different expression patterns of identified lncRNA genes suggest potential function in AIS pathogenesis, direct supporting evidence is lacking. Second, only four pairs of samples were used in microarray analysis. This may lose some important information and decrease the accuracy of biomarker selection. Third, RNA expression in peripheral blood was tested in our study. Regarding the idiopathic scoliosis being a musculoskeletal disease, tissue sample from musculoskeletal system may be more ideal targets. To accurately and comprehensively elucidate the role of lncRNAs in AIS, more comprehensive studies and laboratory and clinical researches are needed.

In summary, to the best of our knowledge, this is the first study that describes the expression profiles of human lncRNAs in AIS using microarray. Altered lncRNAs may play a potential role in the pathogenesis and/or development of this musculoskeletal disease. More work will be needed to confirm whether these lncRNAs play an essential role in the pathogenesis, treatment, and prognosis of AIS.

\section{Conflict of Interests}

The authors declare that there is no conflict of interests regarding the publication of this paper. 


\section{Authors' Contribution}

Xiao-Yang Liu and Liang Wang contributed equally to this work.

\section{Acknowledgment}

The work was supported by the National Natural Science Foundation of China under Grant nos. 81171770, 81372007, and 81272054 .

\section{References}

[1] J. Ogilvie, "Adolescent idiopathic scoliosis and genetic testing," Current Opinion in Pediatrics, vol. 22, no. 1, pp. 67-70, 2010.

[2] E. J. Rogala, D. S. Drummond, and J. Gurr, "Scoliosis: incidence and natural history. A prospective epidemiological study," Journal of Bone and Joint Surgery-Series A, vol. 60, no. 2, pp. 173-176, 1978.

[3] M. O. Andersen, K. Thomsen, and K. O. Kyvik, "Adolescent idiopathic scoliosis in twins: a population-based survey," Spine, vol. 32, no. 8, pp. 927-930, 2007.

[4] C. A. Wise, X. Gao, S. Shoemaker, D. Gordon, and J. A. Herring, "Understanding genetic factors in idiopathic scoliosis, a complex disease of childhood," Current Genomics, vol. 9, no. 1, pp. 51-59, 2008.

[5] N. H. Miller, "Genetics of familial idiopathic scoliosis," Clinical Orthopaedics and Related Research, vol. 462, pp. 6-10, 2007.

[6] N. H. Miller, C. M. Justice, B. Marosy et al., "Identification of candidate regions for familial idiopathic scoliosis," Spine, vol. 30, no. 10, pp. 1181-1187, 2005.

[7] S. Sharma, X. Gao, D. Londono et al., "Genome-wide association studies of adolescent idiopathic scoliosis suggest candidate susceptibility genes," Human Molecular Genetics, vol. 20, no. 7, pp. 1456-1466, 2011.

[8] T. Esposito, R. Uccello, R. Caliendo et al., "Estrogen receptor polymorphism, estrogen content and idiopathic scoliosis in human: a possible genetic linkage," Journal of Steroid Biochemistry and Molecular Biology, vol. 116, no. 1-2, pp. 56-60, 2009.

[9] J. Wu, Y. Qiu, L. Zhang, Q. Sun, X. Qiu, and Y. He, "Association of estrogen receptor gene polymorphisms with susceptibility to adolescent idiopathic scoliosis," Spine, vol. 31, no. 10, pp. 11311136, 2006.

[10] Y. Takahashi, M. Matsumoto, T. Karasugi et al., "Lack of association between adolescent idiopathic scoliosis and previously reported single nucleotide polymorphisms in MATN1, MTNR1B, TPH1, and IGF1 in a Japanese population," Journal of Orthopaedic Research, vol. 29, no. 7, pp. 1055-1058, 2011.

[11] N. L. S. Tang, H. Y. Yeung, K. M. Lee et al., "A relook into the association of the estrogen receptor $\alpha$ gene (PvuII, XbaI) and adolescent idiopathic scoliosis: a study of 540 Chinese cases," Spine, vol. 31, no. 21, pp. 2463-2468, 2006.

[12] R. W. Carthew and E. J. Sontheimer, "Origins and mechanisms of miRNAs and siRNAs," Cell, vol. 136, no. 4, pp. 642-655, 2009.

[13] I. I. G. M. van de Vondervoort, P. M. Gordebeke, N. Khoshab et al., "Long non-coding RNAs in neurodevelopmental disorders," Frontiers in Molecular Neuroscience, vol. 6, article 53, 2013.

[14] C. A. Klattenhoff, J. C. Scheuermann, L. E. Surface et al., "Braveheart, a long noncoding RNA required for cardiovascular lineage commitment," Cell, vol. 152, no. 3, pp. 570-583, 2013.
[15] R. J. Taft, K. C. Pang, T. R. Mercer, M. Dinger, and J. S. Mattick, "Non-coding RNAs: regulators of disease," Journal of Pathology, vol. 220, no. 2, pp. 126-139, 2010.

[16] T. Gutschner, M. Hämmerle, M. Eißmann et al., "The noncoding RNA MALAT1 is a critical regulator of the metastasis phenotype of lung cancer cells," Cancer Research, vol. 73, no. 3, pp. 1180-1189, 2013.

[17] D. G. Weber, G. Johnen, S. Casjens et al., "Evaluation of long noncoding RNA MALAT1 as a candidate blood-based biomarker for the diagnosis of non-small cell lung cancer," BMC Research Notes, vol. 6, no. 1, article 518, 2013.

[18] D. Li, G. Chen, J. Yang et al., "Transcriptome analysis reveals distinct patterns of long noncoding RNAs in heart and plasma of mice with heart failure," PLoS ONE, vol. 8, Article ID e77938, 2013.

[19] M. Stuss, P. Rieske, A. Cegłowska et al., "Assessment of OPG/ RANK/RANKL gene expression levels in peripheral blood mononuclear cells (PBMC) after treatment with strontium ranelate and ibandronate in patients with postmenopausal osteoporosis," Journal of Clinical Endocrinology and Metabolism, vol. 98, no. 5, pp. E1007-E1011, 2013.

[20] R. Nowak, J. Szota, and U. Mazurek, "Vitamin D Receptor gene (VDR) transcripts in bone, cartilage, muscles and blood and microarray analysis of vitamin D responsive genes expression in paravertebral muscles of Juvenile and Adolescent Idiopathic Scoliosis patients," BMC Musculoskeletal Disorders, vol. 13, article 259, 2012.

[21] S. L. Weinstein, L. A. Dolan, J. C. Cheng, A. Danielsson, and J. A. Morcuende, "Adolescent idiopathic scoliosis," The Lancet, vol. 371, no. 9623, pp. 1527-1537, 2008.

[22] J. L. Rinn, M. Kertesz, J. K. Wang et al., "Functional demarcation of active and silent chromatin domains in human HOX loci by noncoding RNAs," Cell, vol. 129, no. 7, pp. 1311-1323, 2007.

[23] M. Guttman, I. Amit, M. Garber et al., "Chromatin signature reveals over a thousand highly conserved large non-coding RNAs in mammals," Nature, vol. 458, no. 7235, pp. 223-227, 2009.

[24] A. M. Khalil, M. Guttman, M. Huarte et al., "Many human large intergenic noncoding RNAs associate with chromatin-modifying complexes and affect gene expression," Proceedings of the National Academy of Sciences of the United States of America, vol. 106, no. 28, pp. 11667-11672, 2009.

[25] U. A. Ørom, T. Derrien, M. Beringer et al., "Long noncoding RNAs with enhancer-like function in human cells," Cell, vol. 143, no. 1, pp. 46-58, 2010.

[26] R. G. Burwell, "Aetiology of idiopathic scoliosis: current concepts," Pediatric Rehabilitation, vol. 6, no. 3-4, pp. 137-170, 2003.

[27] Z. Yin, D. Guan, Q. Fan et al., "LncRNA expression signatures in response to enterovirus 71 infection," Biochemical and Biophysical Research Communications, vol. 430, no. 2, pp. 629-633, 2013.

[28] F. Yang, L. Zhang, X.-S. Huo et al., "Long noncoding RNA high expression in hepatocellular carcinoma facilitates tumor growth through enhancer of zeste homolog 2 in humans," Hepatology, vol. 54, no. 5, pp. 1679-1689, 2011.

[29] F. Yang, L. Zhang, X.-S. Huo et al., "Long noncoding RNA high expression in hepatocellular carcinoma facilitates tumor growth through enhancer of zeste homolog 2 in humans," Hepatology, vol. 54, no. 5, pp. 1679-1689, 2011.

[30] M. Cesana, D. Cacchiarelli, I. Legnini et al., "A long noncoding RNA controls muscle differentiation by functioning as a competing endogenous RNA," Cell, vol. 147, pp. 358-369, 2011. 
[31] R. Velázquez-Cruz, H. García-Ortiz, M. Castillejos-López et al., "WNT3A gene polymorphisms are associated with bone mineral density variation in postmenopausal mestizo women of an urban Mexican population: findings of a pathway-based highdensity single nucleotide screening," Age, vol. 36, article 9635, 2014.

[32] T. Nagaoka, R. Ohashi, A. Inutsuka et al., "The Wnt/planar cell polarity pathway component Vangl2 induces synapse formation through direct control of N-cadherin," Cell Reports, vol. 6, pp. 916-927, 2014.

[33] J. N. Collins, B. J. Kirby, J. P. Woodrow et al., "Lactating Ctcgrp nulls lose twice the normal bone mineral content due to fewer osteoblasts and more osteoclasts, whereas bone mass is fully restored after weaning in association with up-regulation of Wnt signaling and other novel genes," Endocrinology, vol. 154, no. 4, pp. 1400-1413, 2013.

[34] Y. Liu, W.-K. Zheng, W.-S. Gao, Y. Shen, and W.-Y. Ding, "Function of TGF-beta and p38 MAKP signaling pathway in osteoblast differentiation from rat adipose-derived stem cells," European Review for Medical and Pharmacological Sciences, vol. 17, no. 12, pp. 1611-1619, 2013.

[35] C.-C. Niu, S.-S. Lin, L.-J. Yuan et al., "Hyperbaric oxygen treatment suppresses MAPK signaling and mitochondrial apoptotic pathway in degenerated human intervertebral disc cells," Journal of Orthopaedic Research, vol. 31, no. 2, pp. 204-209, 2013.

[36] K. Plath, S. Mlynarczyk-Evans, D. A. Nusinow, and B. Panning, "Xist RNA and the mechanism of X chromosome inactivation," Annual Review of Genetics, vol. 36, pp. 233-278, 2002.

[37] M. V. Koerner, F. M. Pauler, R. Huang, and D. P. Barlow, "The function of non-coding RNAs in genomic imprinting," Development, vol. 136, no. 11, pp. 1771-1783, 2009.

[38] Z. Zhang and D. S. Gilmour, "Pcf11 is a termination factor in Drosophila that dismantles the elongation complex by bridging the CTD of RNA polymerase II to the nascent transcript," Molecular Cell, vol. 21, no. 1, pp. 65-74, 2006.

[39] T. J. Loya, T. W. O'Rourke, and D. Reines, "A genetic screen for terminator function in yeast identifies a role for a new functional domain in termination factor Nab3," Nucleic Acids Research, vol. 40, no. 15, pp. 7476-7491, 2012.

[40] M. Rougemaille, G. Dieppois, E. Kisseleva-Romanova et al., "THO/Sub2p Functions to Coordinate 3/-End Processing with Gene-Nuclear Pore Association," Cell, vol. 135, no. 2, pp. 308$321,2008$.

[41] Z. Zhang, A. Klatt, A. J. Henderson, and D. S. Gilmour, "Transcription termination factor Pcfll limits the processivity of Pol II on an HIV provirus to repress gene expression," Genes \& Development, vol. 21, no. 13, pp. 1609-1614, 2007.

[42] J. S. Hoashi, P. J. Cahill, J. T. Bennett, and A. F. Samdani, "Adolescent scoliosis classification and treatment," Neurosurgery Clinics of North America, vol. 24, no. 2, pp. 173-183, 2013.

[43] G. Qiu, J. Zhang, Y. Wang et al., "A new operative classification of idiopathic scoliosis: a Peking Union Medical College method," Spine, vol. 30, no. 12, pp. 1419-1426, 2005.

[44] Y. Smorgick, Y. Mirovsky, K. C. Baker, Y. Gelfer, E. Avisar, and Y. Anekstein, "Predictors of back pain in adolescent idiopathic scoliosis surgical candidates," Journal of Pediatric Orthopaedics, vol. 33, no. 3, pp. 289-292, 2013.

[45] J. R. Prensner, M. K. Iyer, O. A. Balbin et al., "Transcriptome sequencing across a prostate cancer cohort identifies PCAT1 , an unannotated lincRNA implicated in disease progression," Nature Biotechnology, vol. 29, no. 8, pp. 742-749, 2011. 

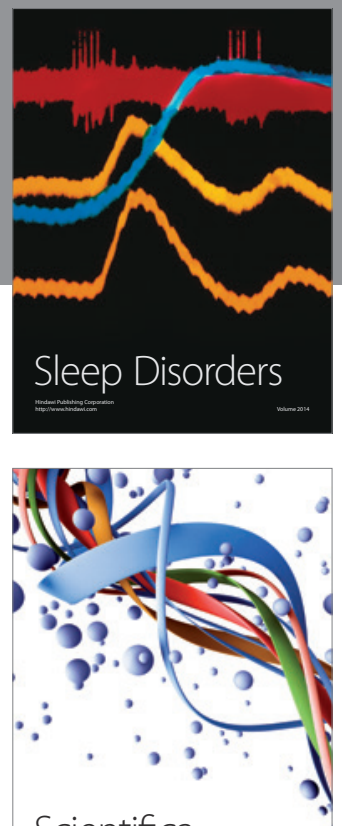

Scientifica
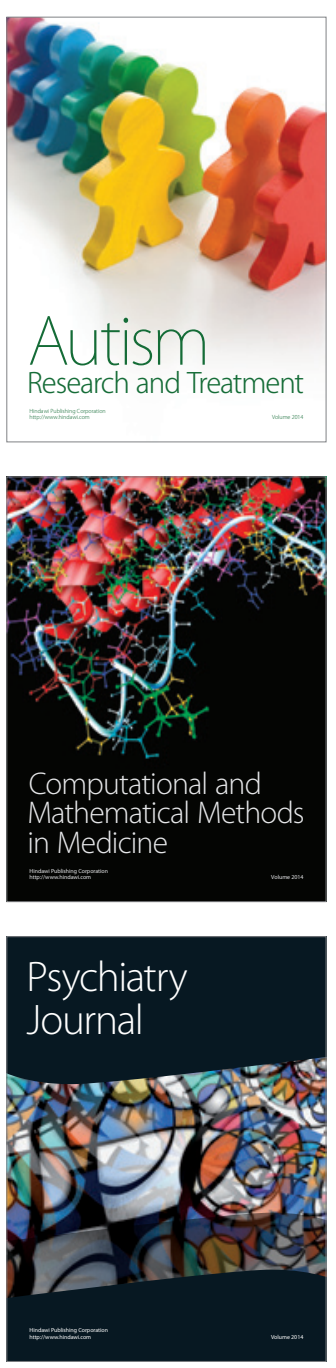
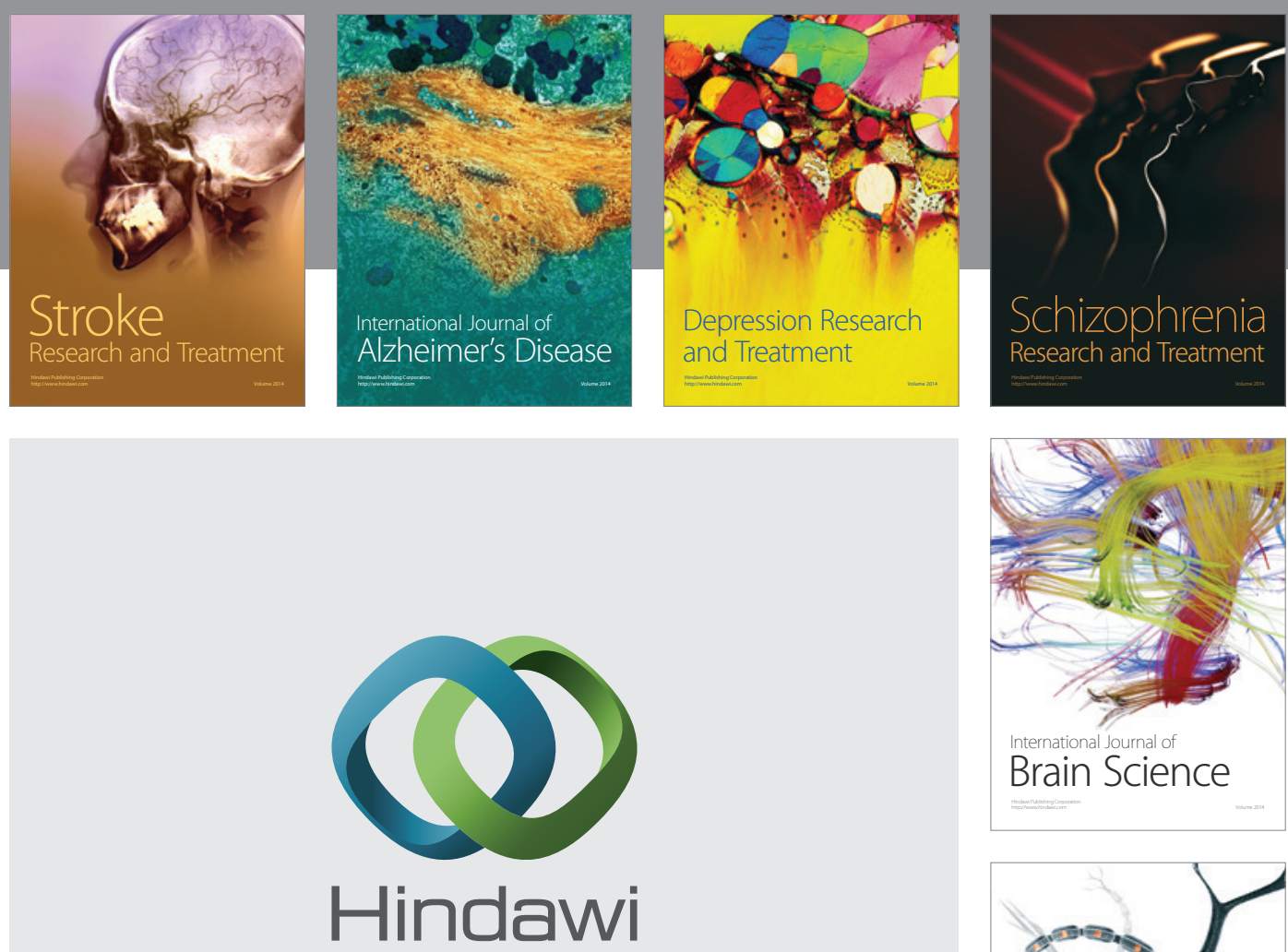

Submit your manuscripts at

http://www.hindawi.com
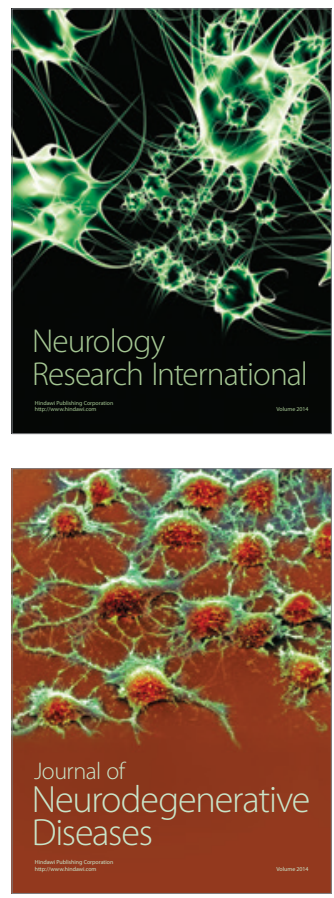

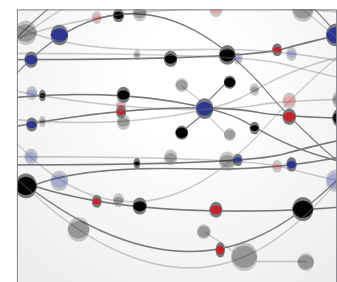

The Scientific World Journal
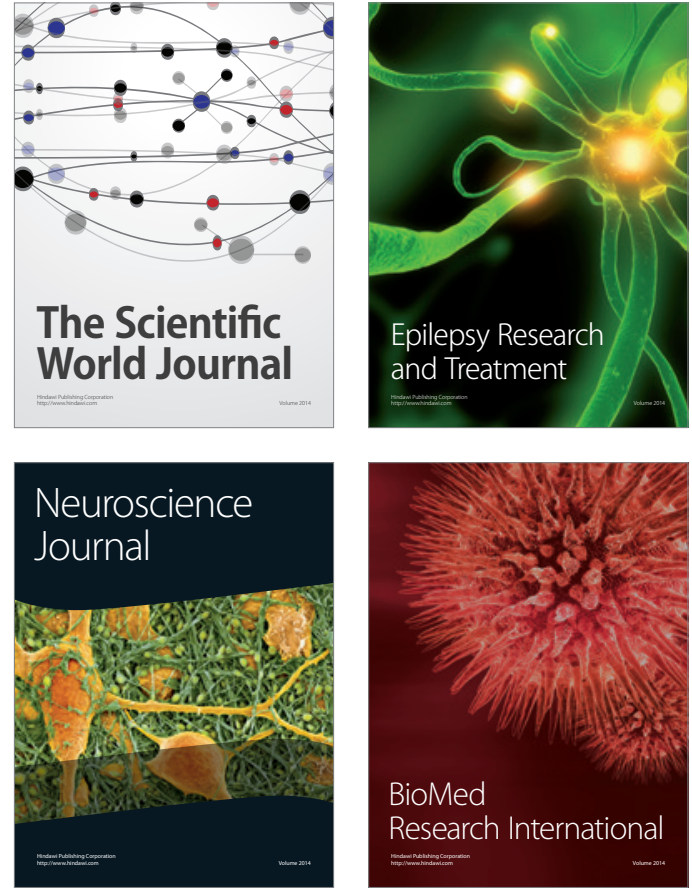

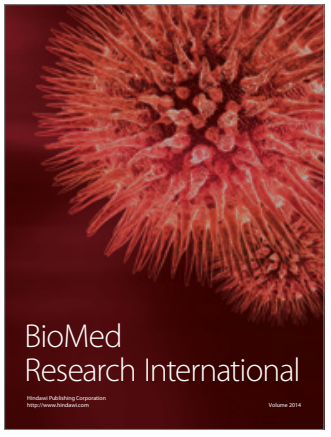

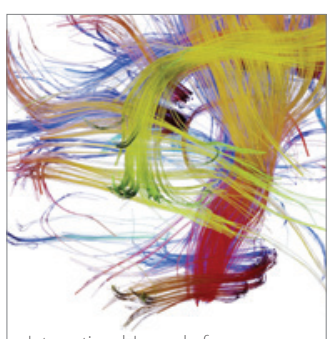

Brain Science

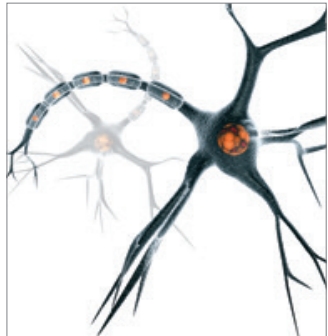

Neural Plasticity
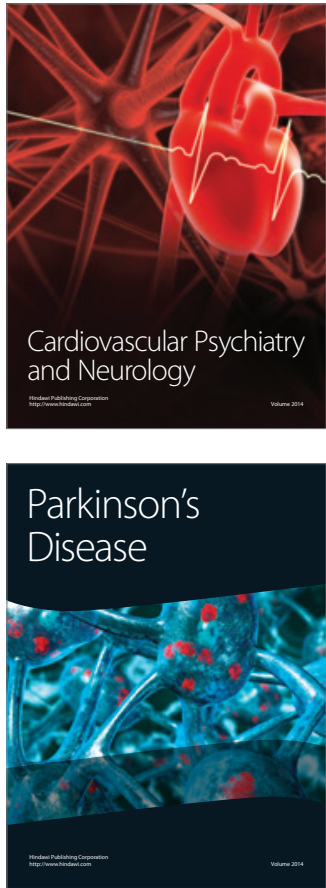